\title{
Síndrome del túnel carpiano de origen secundario
}

\author{
G. Celester Barreiro(1), A. Castro Río(2) ${ }^{(2)}$ J. L. Seohne FreiJido $^{(2)}$ \\ Servicio de Cirugía de la Mano del Hospital Modelo. Mutua la Fraternidad. a Coruña \\ (1) ESPECIALISTA EN CIRUGÍA ORTOPÉDICA Y TRAUMATOLOGÍA \\ (2) MEDICINA ASISTENCIAL
}

Correspondencia

Dr. Gabriel Celester Barreiro

Avda. del Ejército 12, bajo (Mutua)

15006 A Coruña

gcelester@fraternidad.com

Objetivo: El objetivo de este trabajo es investigar la frecuencia de casos de síndrome del túnel carpiano de origen secundario, bien debidos a patología conocida previamente al acto quirúrgico o a anomalías descubiertas en el curso del mismo.

Material y método: Se han revisado retrospectivamente todos los síndromes del túnel carpiano operados por los autores en los últimos 11 años naturales (1 de enero de 1997 al 31 de diciembre de 2007), en los que fueron intervenidas 317 muñecas en 273 pacientes, 44 bilaterales $(13,9 \%)$.

Resultados: Describimos 57 casos (18\%) de patología previa o hallazgos inesperados y comparamos los hallazgos con la bibliografía especializada.

Conclusiones: Las causas más frecuentes de síndrome del túnel carpiano secundario fueron la sinovitis de los tendones flexores y las fracturas mal consolidadas de la extremidad distal del radio. Es probable que las anomalías anatómicas, bien sean musculares, nerviosas o vasculares, actúen como un factor coadyuvante o agravante, pero no sean la causa primaria.

Palabras clave: síndrome del túnel carpiano, síndrome del túnel carpiano de origen secundario, sinovitis de tendones flexores, músculos accesorios mano.
Objective: The objective of this study is to research the frequency of carpal tunnel syndrome cases of secondary origin, either due to pathology known previous to the surgery or to irregularities found during the surgery.

Material and method: We have reviewed all the patients with carpal tunnel syndrome whose surgery we carried out in the last 11 years (January 1 st of to December 31st of 2007). We operated 317 wrist on 273 patients, 44 bilateral $(13,9 \%)$.

Results: We describe the 57 cases $(18 \%)$ of previous pathology or unexpected findings in the 317 treated wrists. We compare our findings with the specialized literature.

Conclusions: The most common causes of secondary carpal tunnel syndrome are the flexor tendons synovitis and the malunion of fractures of the distal end of the radius. It is probable that the anatomic anomalies, be they muscular, nervous or vascular, are just a coadjuvant or worsening factor but not the primary cause.

Key words: carpal tunnel syndrome, secondary carpal tunnel syndrome, flexor tendons synovitis, accessory muscles of the hand. 


\section{INTRODUCCIÓN}

1 un cuando las causas de compresión del nervio mediano en su paso por la cara anterior del carpo son múltiples, el síndrome del túnel carpiano (STC), en cuanto a su etiología, se divide clásicamente en dos grandes grupos: los de causa desconocida, llamados primarios o idiopáticos, y los que son debidos a la existencia, previamente conocida o descubierta en el curso de la intervención quirúrgica, de procesos que disminuyen la luz del túnel extrínsecamente (consolidaciones viciosas, luxaciones inveteradas, etc.) o intrínsecamente por ocupar espacio en su interior (sinovitis, tumores, anomalías anatómicas) con el consiguiente aumento de la presión intracanalicular en ambos casos. Este último grupo forman los STC secundarios.

No abundan las publicaciones sobre grandes series de STC de origen secundario, pero seguramente esta parquedad bibliográfica no refleja su alta frecuencia. En una revisión bibliográfica de los últimos 20 años de la Revista Iberoamericana de Cirugía de la Mano y de la Revista de Ortopedia y Traumatología apenas hemos encontrado comunicaciones. Sin embargo algunos estudios ${ }^{1}$ muestran que hasta un $38 \%$ de los STC pueden ser debidos a patología secundaria.

\section{OBJETIVO}

Son múltiples los condicionantes y procesos patológicos que se han relacionado con el STC: actividad laboral, edad, género, diabetes, fracturas, etc.

El objetivo de este trabajo es analizar cuantos STC operados son idiopáticos y cuantos tienen una causa previamente conocida o presentan en la intervención quirúrgica hallazgos anatómicos, bien sean patológicos o variantes de la normalidad, que ocupan espacio dentro del túnel y puedan estar relacionados con la aparición del síndrome.

\section{MATERIAL Y MÉTODO}

Se analizaron de forma retrospectiva todos los STC operados en esta serie personal desde el 1 de enero de 1997 hasta el 31 de diciembre de 2007 (11 años naturales) en un total de 317 muñecas en 273 pacientes; 44 bilaterales $(13,9 \%)$.

Técnica: Cuando habían sido diagnosticados inicialmente como idiopáticos se realizó una apertura longitudinal palmar corta sobre el ligamento anular volar del carpo en el eje del $4^{\circ}$ dedo sin sobrepasar proximalmente el pliegue flexor de la muñeca. La incisión fue ampliada cuando los hallazgos intraoperatorios o una patología ya conocida lo aconsejaban. En ningún caso se practicó cirugía endoscópica. Todos fueron operados con manguito de isquemia, no se realizó hemostasia, se puso un drenaje de Penrose durante 24-48 horas, se suturó sólo la piel con nylon monofilamento de $4 / 0$, se colocó un vendaje compresivo y se instó al paciente a mover la mano desde el momento en que se le despertase. Se les dice que cada cinco o diez minutos deben abrirla varias veces con fuerza, como para medir por palmos, y cerrarla con fuerza formando un puño con el fin de que el sangrado salga a través del drenaje. Se les prohíbe mojar la herida y se insiste en el uso de la mano desde el primer momento para comer, calzarse, vestirse, etc., procurando minimizar el acto operatorio y explicándole a los pacientes que las peores complicaciones de esta intervención vienen por el desuso.

El empleo de un drenaje y la movilización precoz tienen un mismo cometido: procurar que salga todo el sangrado que se produzca con el fin de evitar hematomas dentro del túnel y con ello la fibrosis. Por su dureza y forma acanalada, la mitad longitudinal de un drenaje de Penrose pequeño ha demostrado ser el más eficaz tras varios probados. Con materiales más finos, como una tira de guante quirúrgico, se colapsa el ojal cutáneo; el Redón aspirativo prolonga la estancia hospitalaria y a menudo pierde el cometido por oclusión de los poros. No se utilizó coagulación térmica para no quemar terminaciones sensitivas y disminuir así las molestias del postoperatorio. Tanto la incisión cutánea como la efectuada sobre el ligamento anular son cortas, longitudinales y producen mínimas secciones vasculares, por lo que el sangrado suele ser escaso; las gasas apenas están manchadas de sangre cuando se retira el drenaje. 
Anestesia: Se operaron 301 manos con anestesia regional (243 axilar y 58 regional endovenosa), 10 con anestesia general y 6 con anestesia local.

Edad: Las edades oscilaban entre 21 y 84 años; con una media de 45,6 años

Género: 212 mujeres $(77,7 \%), 61$ hombres $(22,3 \%)$.

Complicaciones: No se registró ningún caso de lesión yatrógena, vascular o nerviosa, ni de infección. Tampoco ningún síndrome de dolor regional complejo postoperatorio, probablemente por la insistencia en aconsejar el uso precoz de la mano.

La complicación más frecuente que registra la bibliografía consultada es la persistencia de síntomas tras la intervención. La hemos observado en algunas mujeres, nunca en hombres, $y$ rara vez requiere tratamiento. En mujeres jóvenes cuyo síndrome compresivo es de meses o muy pocos años de evolución y tiene una clara relación con una actividad de fuerza (carniceras, charcuteras, etc.), los síntomas suelen desaparecer paulatinamente en pocas semanas tras la intervención quirúrgica. Por el contrario, en algunas mujeres añosas con largos procesos evolutivos a veces permanecen los síntomas pero son más tolerables que antes de la cirugía y, por lo general, las pacientes se muestran satisfechas. Hasta donde sabemos, en esta serie sólo una paciente de 60 años, cajera de supermercado, precisó ser reintervenida por este motivo. En la revisión se apreció una notoria fibrosis perineural. Se liberó ampliamente el nervio y se cubrió con una bandeleta del ligamento anular, pese a lo cual persistieron las molestias y la paciente no se reincorporó al trabajo.

\section{RESULTADOS}

Fueron encontradas anomalías inesperadas o patología previa ya conocida en 57 manos (18\% de las operadas), dividiéndose estos hallazgos del siguiente modo:

\section{Sinovitis de flexores: 32}

Inespecíficas (mecánicas o seronegativas): 25

Reumáticas: 5 (3 artritis reumatoide, 1 lúpica, 1 úrica)

Sépticas: 2 (tuberculosas)
Tumores: 3

Xantogranuloma de vainas: 1

Ganglión: 1

Leiomioma: 1

Patología ósea: 10

Secuelas de fractura de la extremidad distal del radio: 7

Luxación inveterada del semilunar: 1

Disociación escafolunar antigua: 1

Calcificación en la cara anterior del carpo: 1

Anomalías vasculares: 2

Arteria mediana: 2

\section{Anomalías nerviosas: 4}

Nervio mediano bífido: 4 (en 3 pacientes; 1 bilateral)

Anomalías musculares: 6

Vientres musculares de flexores dentro del túnel: 3 (en 2 pacientes)

Lumbrical dentro del túnel: 1

Palmaris profundus: 2

\section{DISCUSIÓN}

Género y edad: La proporción de hombres (22,3\% del total), así como la edad media algo baja y la menor proporción de casos bilaterales que en otras series se debe, probablemente, a que en los últimos 7 años desarrollamos gran parte de nuestra actividad en el ambiente laboral, donde la población masculina predomina sobre la femenina con respecto a la población general.

La comparación de estos parámetros, género y edad, debe hacerse entre poblaciones homogéneas, por lo que en este estudio no tienen un valor estadístico significativo. Sí parece relevante el hecho de que de los 61 hombres, 18 $(29,5 \%)$ presentaran patología previa o hallazgos ocasionales, mientras que éstos sólo se encuentran en 37 de las 212 mujeres $(17,4 \%)$. Lo que indica claramente que el STC idiopático es más frecuente en mujeres y el STC secundario en hombres.

Sinovitis de los tendones flexores: Continúa actualmente en discusión el factor desencadenante de la sinovitis de los flexores. Cada vez tiene más adeptos la teoría vascular ${ }^{2,3}$, se- 
gún la cual la sinovitis de los flexores es consecuencia del síndrome compresivo y no su causa, debido a que el aumento de presión dentro del túnel provoca una isquemia en los vasos epineurales y en los del paratendón de los flexores. Esta hipoxia aumenta la permeabilidad del endotelio de los capilares produciéndose una salida de proteínas plasmáticas y células leucocitarias que en las vainas tendinosas causaría un aumento de su grosor. Para algunos autores ${ }^{4}$ la relación entre la tenosinovitis y la afectación nerviosa en realidad no sería tanto de causaefecto como dos procesos concomitantes, pues el aumento de presión intracanalicular que produce los cambios degenerativos en la sinovial tendinosa, conlleva similares cambios fibróticos en el tejido perineural. Sin embargo, aun cuando numerosos estudios parecen demostrar este aspecto, la teoría isquémica no explica el motivo de que aumente la presión dentro del túnel en pacientes jóvenes y varones, mientras que sí lo hace la teoría mecánica al sugerir que la utilización de la mano en actividades de fuerza, combinada con reiterados movimientos de flexoextensión, produce una isquemia de los tendones flexores conjuntamente con su irritación mecánica, desarrollándose así una inflamación crónica de las vainas que conlleva a su engrosamiento. Esta hipertrofia del paratendón puede verse como una medida protectora para evitar las rupturas tendinosas del mismo modo que se engruesan la sinovial que tapiza la cara interna de las bursas (idéntico tejido histológico) o los tejidos conjuntivo y epitelial en los puntos de rozamiento o hiperpresión.

La teoría mecánica no explica, en cambio, por qué se encuentra sinovitis tendinosa sólo en una pequeña parte de los STC, y no más entre personas jóvenes con trabajos de fuerza que en la población general.

La creencia en el factor mecánico está muy extendida entre los propios trabajadores, médicos de empresa, traumatólogos que ejercen en el ámbito laboral y se refleja asimismo en la legislación española, que considera al STC una enfermedad profesional en aquellas ocupaciones de fuerza o de movimientos repetitivos de la mano. Sin embargo, esta opinión tan difundida no parece suficientemente demostrada para algunos autores. Dias et al. ${ }^{5}$ postulan que el trabajo, tanto en ocupaciones repetitivas como no repetitivas, no es la causa ni aun un factor agravante o acelerador del STC, lo que parece desmentir la experiencia cotidiana de los traumatólogos laborales que encuentran más frecuente la enfermedad entre cajeras de supermercado jóvenes, por ejemplo, que entre mujeres de la misma edad desocupadas o con poca sobrecarga funcional, aun cuando no haya en aquéllas más presencia de tenosinovitis. Discrepamos con Dias et $\mathrm{al}^{5}$, pues aunque es muy difícil diferenciar los oficios por el grado de esfuerzo que requieren sus actividades básicas y habituales, es evidente que un minero debe realizar mayores esfuerzos físicos que un abogado en el ejercicio de sus respectivas profesiones. De los pacientes operados en esta serie, el $28,6 \%$ eran menores de 40 años y de éstos el $83 \%$ tenían ocupaciones que se podrían considerar de esfuerzos moderados o grandes. No obstante, no se encontró en ellos una relación directa entre sinovitis y actividades de fuerza.

Uno de los pacientes intervenidos recientemente era un varón de 24 años, pintor de profesión, que llevaba dos años ininterrumpidos utilizando una pistola de presión y extendiendo pintura pastosa con una llana empleando para ambas actividades su mano derecha, la única con afectación nerviosa. Tanto la historia clínica como las pruebas de Tinel y Phalen y la electromiografía eran inequívocas de un STC. El paciente tuvo una mejoría absoluta tras la intervención. Sin embargo, no se apreció ninguna alteración macroscópica de las vainas tendinosas ni cualquier otro detalle relevante en el interior del túnel y el estudio histológico no mostró ninguna alteración de las vainas sinoviales.

En otros casos en los que sí se aprecia una exuberante sinovitis macroscópica, como en la paciente que muestra la Figura 1A, maestra de 56 años de edad, en la que el nervio mediano de la muñeca izquierda (no dominante) tenía una notoria muesca causada por la sinovitis de los tendones flexores, tampoco se encontraron hallazgos relevantes en el estudio histológico ni en el cultivo de las vainas.

Pickering et al. ${ }^{6}$ realizaron biopsia a las vainas tendinosas de 58 manos operadas en 50 trabajadores varones encontrando que 27 tenían un 


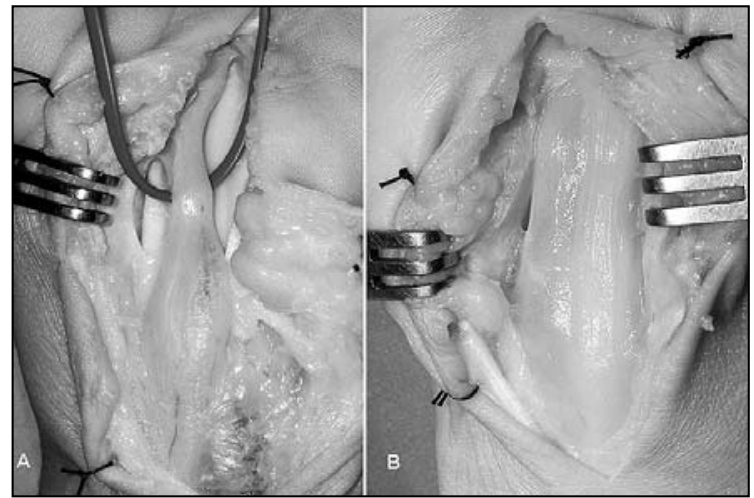

Figura 1. A: STC por sinovitis de los tendones flexores. Se aprecia la marcada impronta en el nervio mediano producida por la presión de las vainas tenosinoviales hipertrofiadas. B: STC por sinovitis lúpica.

engrosamiento de la membrana sinovial igual o mayor de 1 milímetro y 31 no, resultado sin valor significativo y desalentador porque el estudio histológico de estos casos no parece más concluyente que la mera inspección de las vainas por un cirujano experimentado.

Faithfull et al. ${ }^{7}$ encontraron tres tipos de anomalías en el estudio anatomopatológico de las vainas tendinosas estudiadas en 29 pacientes operados de STC. En la mayor parte predominaba el edema y en otras la infiltración de linfocitos o de fibrina; es decir, cambios absolutamente inespecíficos de un proceso inflamatorio en distintos periodos de evolución y perfectamente comparables a los cambios histológicos que hemos apreciado cuando enviamos al laboratorio muestras de vainas de una enfermedad de De Quervain o de dedos en resorte. Kerr et al. ${ }^{8}$ no encontraron signos de inflamación de las vainas en el 96\% de 625 STC idiopáticos biopsiados (a todos los operados les realizó sinovectomía); 601 manos presentaron una sinovitis fibrosa benigna, en el $4 \%$ obtuvo hallazgos de inflamación aguda y en el 0,2\% (1 caso) de inflamación crónica.

Un grave problema para analizar la frecuencia y diferencias cualitativas de las sinovitis es que no existe un criterio unánime entre los patólogos a la hora de valorarlas. Sus informes emplean a menudo una terminología ambigua y confusa para los clínicos, de ahí que los porcentajes entre distintos autores sean tan dispares.

Para explicar la etiopatogenia del STC en personas jóvenes, en cuyo grupo abundan los varones, Lluch $^{2}$ sugiere como causa posible la entrada dinámica de vientres musculares dentro del túnel. Ha sido ampliamente descrito el hallazgo de lumbricales o de vientres musculares de los tendones flexores en el túnel. Nosotros hemos observado ambas anomalías pero no las creemos frecuentes. Cuando operamos pacientes jóvenes pedimos a nuestro ayudante que flexione la muñeca del paciente con la mano cerrada para buscar la presencia de lumbricales y que la extienda con los dedos estirados para ver si entran vientres flexores, y rarísima vez se observan.

Continúa pues en discusión el aspecto fisiopatológico de las tenosinovitis como causa, consecuencia o factor concomitante de la alteración del nervio mediano en el STC. Seguramente se necesitan estudios más amplios en poblaciones muy homogéneas y otros, más difíciles de realizar, con medidas de presión intracanalicular durante la realización de determinadas actividades.

Por otra parte, son comprensibles las dudas que pueda tener la población general con respecto al factor ocupacional en la etiopatogenia del STC, y aun los médicos poco familiarizados con esta patología, cuando ni los propios cirujanos de la mano concordamos en aspectos básicos. Así, mientras algunos encuentran una relación con el uso de teclados, a nosotros nos parece altamente improbable esta causa y menos todavía por el uso de un ratón de ordenador, actividad en que la muñeca está apoyada en posición neutra, no hace flexión, extensión ni fuerza con los tendones flexores, a excepción de la, casi imperceptible, efectuada con el flexor digitorum profundus del índice para deprimir un milímetro un botón que apenas ofrece resistencia y la empleada por el flexor pollicis longus, igual de diminuta, para sujetar el ratón contra la cara radial del $4^{\circ}$ ó $5^{\circ}$ dedos. Usando el ratón con el antebrazo apoyado, la mano sólo hace un pequeño deslizamiento radial (abductor pollicis longus y extensor carpi radialis longus), cubital (flexor carpi ulnaris y extensor carpi ulnaris) y mínimos movimientos de flexoextensión de las metacarpofalángicas con la musculatura intrínseca de la mano; es decir, utilizando tendones que no pasan por el túnel carpiano. 
Weiss ${ }^{9}$ midió la presión intracanalicular en los distintos grados de recorrido articular de la muñeca. Encontró que tanto en los pacientes con STC como en voluntarios sanos la presión era mayor cuanto más se alejaba de la posición neutra, tanto en flexión como en extensión o en desviaciones radial y cubital. La presión menor dentro del túnel se registró entre $7^{\circ}$ de flexión y $11^{\circ}$ de extensión y entre $8^{\circ}$ de desviación radial y $10^{\circ}$ de desviación cubital, y era máxima en la flexión y extensión extremas.

El ratón informático se utiliza prácticamente en la «posición ideal» de la muñeca y ésta nunca hace flexión -no lo permite la mesa- ni extensión, ya que el cursor no funciona si el ratón pierde el contacto con la superficie.

Un reciente estudio sueco ${ }^{10}$ basado en una encuesta a 2465 personas entre 25 y 65 años sugiere que el uso de teclado de ordenador está en relación inversa con la afectación del STC al mostrar que la mayor frecuencia de este síndrome se da en las personas que no utilizan ordenador $(5,2 \%)$, frente a un $4,9 \%$ en quienes lo usan menos de una hora diaria, $2,9 \%$ entre quienes lo usan entre una y cuatro horas y un $2,6 \%$ entre los usuarios de cuatro o más horas diarias.

En lo que sí parece existir acuerdo es que del mismo modo que la epineurectomía no aporta beneficios en el tratamiento del STC, tampoco los tiene la sinovectomía sistemática en los casos de STC idiopáticos ${ }^{2,11}$, debiendo realizarse sólo cuando sea muy notoria la proliferación sinovial.

En esta serie que presentamos, muy heterogénea en cuanto a ocupaciones laborales, en la que se mezclan jubiladas con varones de gran esfuerzo físico, sólo 32 muñecas de 317 presentaban una sinovitis franca desde un punto de vista macroscópico y 8 lo eran por una causa conocida. 25 casos fueron tipificados como de origen mecánico o seronegativas por ser anodino el estudio anatomopatológico, 5 tenían una enfermedad reumática diagnosticada previamente y 2 eran de origen séptico. La Figura $1 B$ muestra un nervio mediano encintado por la compresión del saco sinovial de una tendinitis lúpica.

Los dos casos de sinovitis infecciosas han sido por tuberculosis de las vainas flexoras. La tenosinovitis es la forma de presentación habitual en la infección tuberculosa de la mano y muñeca, siendo la afectación volar dos veces más frecuente que la dorsal y suele diagnosticarse cuando ya produce una compresión del nervio mediano ${ }^{12-15}$.

Tumores: Aun siendo rara la presencia de tumores dentro del túnel carpiano, el nervio mediano puede sufrir compresión por masas de los tejidos limítrofes, por tumores propios del tejido neural (schwannomas, neurofibromas) y por tumores que se originan en los tejidos no neu-
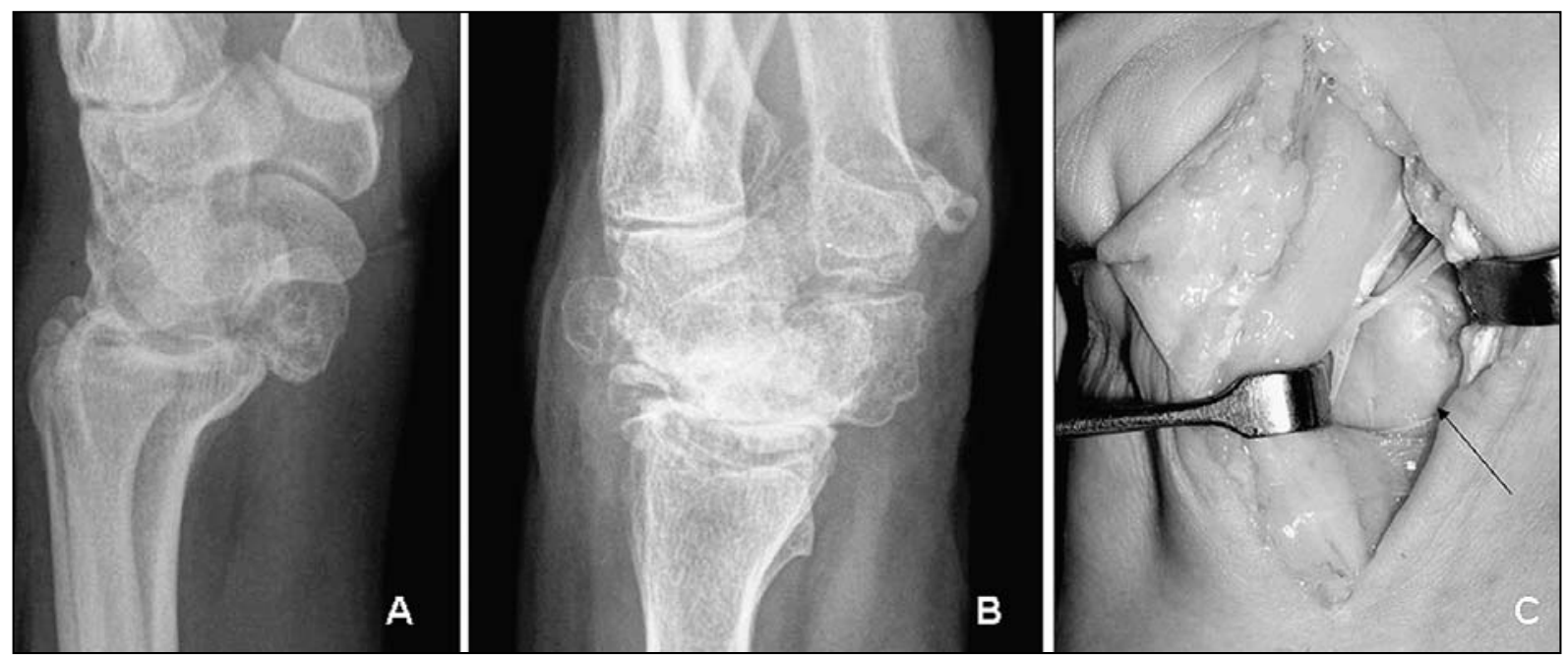

Figura 2. A: STC por luxación del semilunar de 18 años de evolución. B y C: STC por disociación escafolunar antigua (SLAC). El progresivo colapso del carpo desplazó al polo distal del escafoides situándolo bajo el nervio (flecha). 


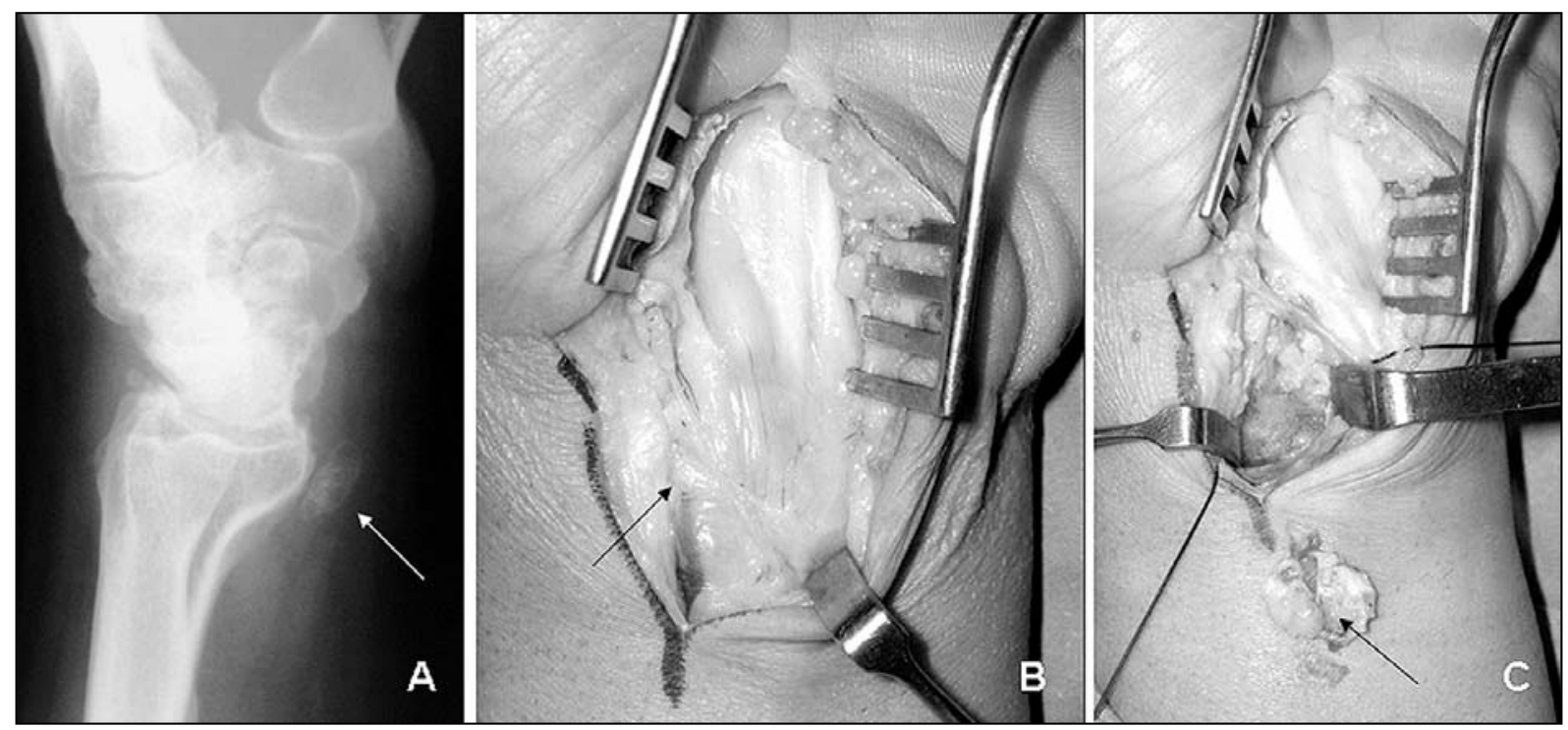

Figura 3. A: STC por calcificación sobre la cara anterior de la epífisis distal del radio. B y C: La masa está situada bajo el nervio mediano y la rama motora tenar.

rales del nervio (lipoma intraneural, ganglión intraneural, hamartoma lipofibromatoso, angioma intraneural) ${ }^{16-18}$.

Tanto en esta serie ( 1 xantogranuloma de vainas, 1 ganglión articular, 1 leiomioma de un lumbrical que penetraba en el túnel por su extremo distal) como en la bibliografía consultada son más frecuentes las masas exoneurales ${ }^{19}$, 20. La exéresis de las mismas suele resolver el cuadro en la mayor parte de los casos.

Patología osteoarticular: El aumento de presión que producen las consolidaciones viciosas de las fracturas de la extremidad distal del radio dentro del canal carpiano, así como las luxaciones carpianas, especialmente la del semilunar (Figura 2A), y los colapsos carpianos (Figura 2B y C), es un factor etiológico bien conocido como responsable del STC ${ }^{21}$. Sin embargo, muy pocas fracturas de la extremidad distal del radio producen un STC duradero, porque, como bien explica $\mathrm{Lluch}^{2}$, estas fracturas no producen una deformidad en la luz del túnel sino a un nivel más proximal, siendo el síndrome compresivo relativamente frecuente en las primeras semanas por el edema y hemorragia fracturaria o por posturas de inmovilización en flexión forzada, pero no al cabo de los años.

En cualquier caso, aun siendo rara, en nuestra experiencia la causa osteoarticular más fre- cuente ha sido la consolidación viciosa de las fracturas distales del radio.

También se han comunicado casos por calcificaciones y depósitos amiloideo, cálcico, úrico, de pirofosfato, etc., si bien estas causas suelen ser excepcionales ${ }^{22,23}$. Un paciente de esta serie presentó un STC producido por una calcificación de origen incierto en una muñeca artrósica pero con la calcificación muy alejada de la interlínea articular (Figura 3).

Anomalías vasculares: El STC provocado por patología vascular es extremadamente raro. Son excepcionales las comunicaciones de casos producidos por hemorragias, aneurismas, trombosis o calcificación de la arteria mediana ${ }^{24}$. Hemos observado la existencia bien desarrollada de esta arteria sólo en dos pacientes y en ambas la consideramos una simple variante de la normalidad sin repercusión en el cuadro clínico. Rus ${ }^{25}$ describe un caso en que la arteria mediana coexiste con un músculo aberrante, accesorio del palmaris longus.

Anomalías nerviosas: Las únicas anomalías halladas en esta serie en el nervio mediano han sido dos casos de bifurcación prematura dentro del canal. Gwynne ${ }^{26}$ comunicó un caso de nervio bífido concomitante con la existencia de un tendón palmaris profundus. Sin unas gafas-lupa ambas estructuras pueden con- 


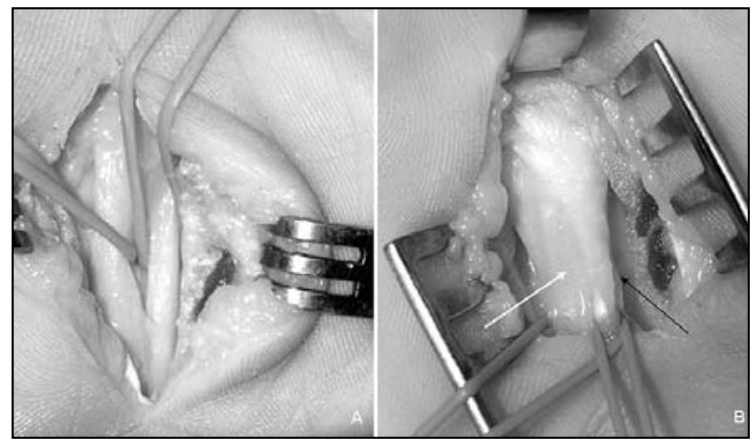

Figura 4. A: Un caso de STC con anomalía nerviosa (mediano bífido). B: Otro con anomalía muscular (tendón de palmaris profundus -flecha negra-englobado en la misma vaina que el nervio mediano -flecha blanca-). Ambas anomalías pueden confundirse fácilmente, de ahí la conveniencia de emplear unas gafas-lupa antes de realizar ninguna resección.

fundirse fácilmente (Figura 4). Es primordial diferenciarlas, pues mientras la resección del tendón accesorio restaría presión al nervio, la resección de cualquier rama nerviosa a ese nivel produciría una desastrosa secuela.

Anomalías musculares: Las anomalías musculares del antebrazo y de la mano no son muy raras y son objeto de frecuentes comunicaciones cuando causan alteraciones nerviosas o vasculares por compresión.

La alteración muscular más frecuente es la presencia de vientres musculares de los flexores dentro del túnel ${ }^{20,27}$ (Figura 5A), seguida por la presencia de lumbricales implantados muy proximalmente ${ }^{20,27,28}$ (Figura 5B y C). Más excepcional es la existencia de un palmaris profundus ${ }^{29,30}$.

Uno de los músculos del antebrazo que presenta mayor número de variedades o anomalías es el palmaris longus ${ }^{31}$. En un estudio sobre 1600 especimenes anatómicos Reimann et al. ${ }^{32}$ encontraron 138 afectaciones de este músculo en 530 anomalías musculares del antebrazo. Sin embargo, es rara y dudosa la participación de este músculo en el STC. Cantero ${ }^{33}$, en su tesis doctoral encuentra 4 alteraciones de este músculo en 58 observaciones de anomalías musculares de la mano y antebrazo, pero en ninguno de sus casos el palmaris longus provocaba un STC. En cambio, si tenían síntomas de compresión del nervio mediano los 5 pacientes en los que halló alteraciones del flexor digitorum superficialis, bien por aumento de número o por penetrar sus vientres musculares dentro del túnel, y en 4 de las 5 manos con alteraciones de los lumbricales, por la misma razón.

Muy pocas veces es posible discernir si las anomalías musculares son un mero hallazgo ca-

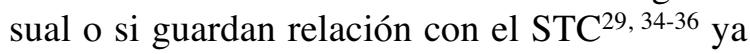
que en la mayor parte de los casos en que aparece un músculo accesorio no se aprecia ninguna depresión del nervio achacable a la presión tendinosa. Rara vez el nervio presenta una cla-

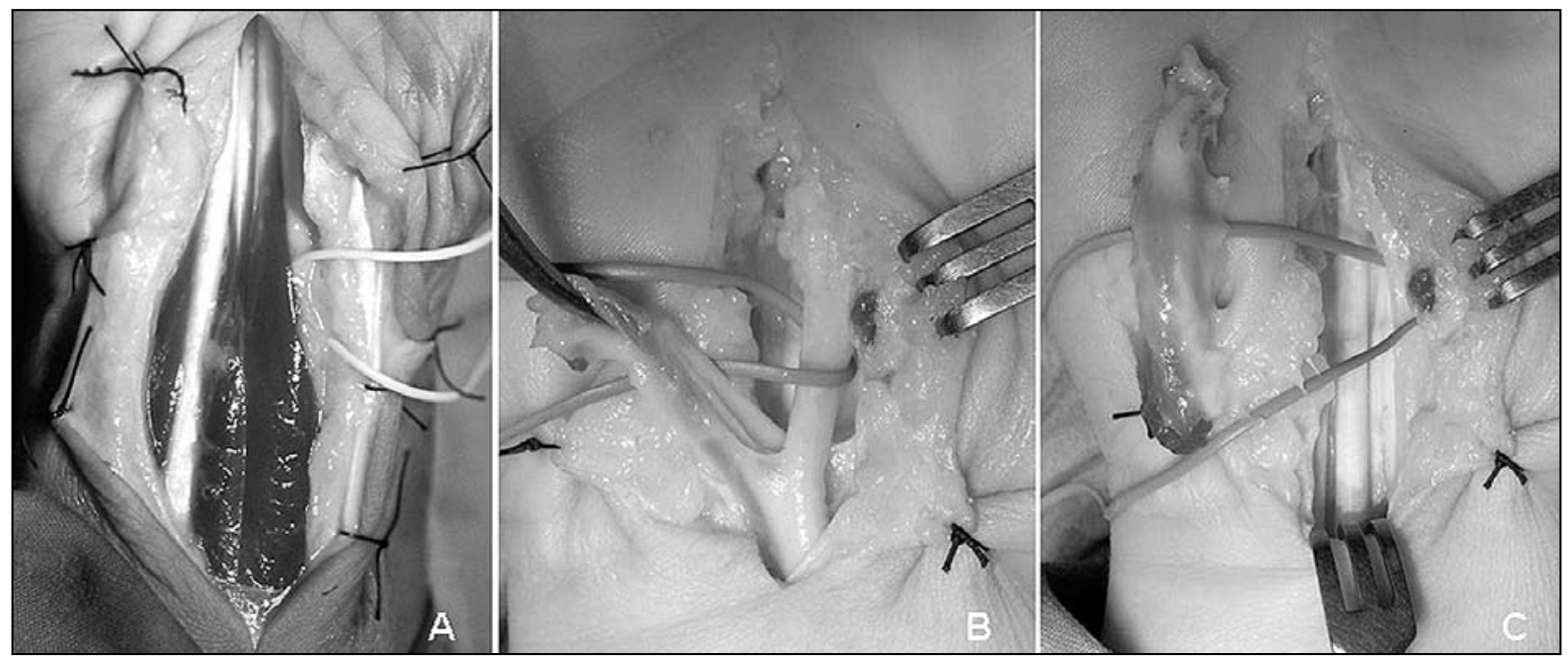

Figura 5. A: Vientres musculares de los tendones flexores dentro del túnel. B y C: Lumbrical anómalo insertado en los tendones flexores superficiales -no en los profundos, como es normal-del $2^{\circ}$ y $3^{\circ}$ dedos, con la fascia firmemente adherida al epineuro. 
ra muesca en la zona de contacto con el tendón ${ }^{37}$; de hecho, muchos pacientes de los descritos en la literatura médica con palmaris profundus comenzaron con síntomas por encima de los 50 años de edad ${ }^{37-39}$.

En rigor, el hallazgo de una anomalía anatómica determinada no es una cuestión de azar, sino de estadística, y por lo general las grandes series aportan datos semejantes. Sin embargo, la revisión de la bibliografía especializada muestra que no siempre es fácil establecer qué es normal y qué no lo es dentro del túnel carpiano incluso para cirujanos experimentados. En esta serie hemos encontrado 6 anomalías musculares en 317 túneles $(1,9 \%)$, cifras semejantes a las obtenidas por Delgado et al. ${ }^{27}(2,5 \%)$ y muy dispares a las de otros autore ${ }^{20}$ que triplican el número de hallazgos $(6 \%)$ al incluir 8 casos de opponens digiti minimi dentro del túnel en $235 \mathrm{mu}$ ñecas operadas, mientras que la serie de Delgado et al. ${ }^{27} \mathrm{y}$ la nuestra no registran ninguno en un total de 832 muñecas, sumadas ambas. Puesto que el opponens digiti minimi se inserta en el hueso ganchoso y en la parte inferior e interna del ligamento anular, es normal encontrar fibras musculares en la parte más distal y cubital del túnel. Anomalías de este músculo y del $a b d u c$ tor digiti minimi en sus inserciones proximal y distal han sido referidas como causa de compresión del nervio cubital ${ }^{33,39}$ pero no encontramos publicaciones relacionándolo con el STC. Dado las numerosas variaciones de inserción de algunos músculos es a menudo controvertido establecer cuáles son normales y cuáles no.

Con respecto al tratamiento del STC, la controversia entre el acceso a cielo abierto versus vía endoscópica sigue vigente en la actualidad. Teniendo en cuenta el significativo número de hallazgos apreciables con un abordaje suficientemente amplio, y que grandes series de des- compresión endoscópica o por vías mínimamente invasivas ${ }^{40-43}$ no registran ni un solo hallazgo dentro del canal, creemos que aparte de sus numerosas contraindicaciones ${ }^{44}$ la crítica más sólida al método endoscópico es que no permite una completa exploración del túnel, con lo que muchos casos de STC pueden quedar mal tratados.

\section{CONCLUSIONES}

1. La patología secundaria que ocasiona con mayor frecuencia un STC es, con gran diferencia, la sinovitis de las vainas flexoras $(57 \%$ de todas las causas en esta serie). Predominan las de origen incierto con el $75 \%$ de los casos frente al $25 \%$ de las tendinitis infecciosas o por enfermedad reumática conocida.

2. La causa osteoarticular más frecuente fue la consolidación viciosa de fracturas de la extremidad distal del radio.

3. Probablemente las anomalías anatómicas nerviosas (nervio bífido) o vasculares (presencia de arteria mediana) encontradas al abrir el túnel carpiano no guarden relación con el síndrome compresivo, mientras que las anomalías musculares sí puedan ser un factor agravante o causa primaria del STC dependiendo del volumen que ocupen.

4. La alta incidencia de patología oculta (sinovitis, tumores, etc.) o anomalías anatómicas dentro del túnel justifica siempre revisar todo su interior y el aspecto del nervio en busca de posibles causas de compresión.

Agradecimiento. A la Srta. Raquel López Rodríguez, DUE, por su valiosa colaboración en la recogida de datos estadísticos. 


\section{BIBLIOGRAFÍA}

1. De Pablos J, Artieda J, Cañadell $J$ et al. El estudio clínico y preclínico en el síndrome el túnel carpiano. Rev Ortop Traum, 1986; 30 IB: 7-15.

2. Lluch Homedes A. El síndrome del túnel carpiano. Edit. Mitre: Barcelona, 1987.

3. Lluch Homedes A. Thickening of the synovium of the digital flexor tendons: cause or consequence of the carpal tunnel syndrome. J. Hand Surg $\mathrm{Br}$, 1992; 17: 209-12.

4. Sud V, Tucci MA, Freeland AE et al. Absortive properties of sinovium harvested from the carpal tunnel. Microsurgery, 2002; 22: 316-9.

5. Dias JJ, Burke FD, Wildin CJ et al. Carpal tunnel syndrome and work. J. Hand Surg $\mathrm{Br}$, 2004; 29: 329-33.

6. Pickering SAW, Stevens A, Davis TRC. Work practices histopathological changes in the tenosynovium in carpal tunnel síndrome in men. $\mathrm{J}$ Hand Surg $\mathrm{Br}, 2004$; 29: 325-8.

7. Faithfull DK, Moir DH, Ireland J. The micropathology of the typical carpal tunnel syndrome. J Hand Surg Br, 1986; 11: 131-2.

8. Kerr ChD, Sybert DR, Albarracín NS. An analysis of the flexor synovium in idiopathic carpal tunnel syndrome: report of 625 cases. J Hand Surg Am, 1992; 17: 1028-30.

9. Weiss ND, Gordon L, Bloom T. et al. Position of the wrist associated with the lowest carpal-tunnel pressure: Implications for splint design. J Hand Surg Am, 1995; 77: 1695-9.

10. Atroshi I, Gummersson Ch, Ornstein E et al. Carpal tunnel syndrome and keyboard use at work: a population-based study. Arthritis Rheum, 2007; 56: 3620-5.

11. Shum Ch, Parisien M, Strauch $\mathrm{RJ}$ et al. The role of flexor tenosynovectomy in the operative treatment of carpal tunnel syndrome. J Bone Joint Surg Am, 2002; 84: 221-5.
12. Celester Barreiro G, Irisarri Castro C, Enes-Martins A et al. Tuberculosis de la mano. A propósito de una serie de 11 casos. Acta Ortop Gallega, 2005; 1: 10-4.

13. Suso S, Peidro L, Ramón R. Tuberculous synovitis with «rice bodies" presenting as carpal tunnel syndrome. J Hand Surg Am, 1988: 13: 574-6.

14. Lee KE. Tuberculosis presenting as carpal tunnel syndrome. $\mathrm{J}$ Hand Surg Am, 1985; 10, 242-5.

15. Wada A, Nomura S, Ihara F. Mycobacterium kansasii flexor tenosynovitis presenting as carpal tunnel syndrome. J. Bone Joint Surg Br, 2000; 25: 308-10.

16. Ayala H, Lluch A, Irisarri C. Tumores de los nervios periféricos. Rev Esp Cir Mano, 1995; 49: 73-84.

17. Luanco M, Carvajo F, Sánchez M. Hamartoma lipomatoso del nervio mediano. Rev Esp Cir Mano, 1995; 49: 85-8.

18. Nogueira A, Pena C, Martínez $\mathrm{MJ}$ et al. Hyperostotic macrodactyly and lipofibromatous hamartoma of the median nerve associated with carpal tunnel syndrome. Ann Chir Main, 1999; 18: 261-71.

19. Luanco M, Sánchez M, Martínez $\mathrm{A}$ et al. Síndrome del túnel carpiano y resorte en la muñeca producidos por un lipoma de las vainas tendinosas y un músculo lumbrical anómalo. Rev Esp Cir Mano, 1990; 39: 23-6.

20. Dudley Porras AF, González del Pino J, Lovic A et al. Síndrome del túnel carpiano: hallazgos intracanal. Rev Ortop Traum, 1998; 42: 103-9.

21. Gutiérrez Carbonell P, Pérez Aznar A, Lizaur Utrilla A. Estudio de las presiones normales del túnel carpiano en comparación con fracturas articulares de muñeca. Rev Ortop Traum, 1994; 38 IB (Sup II): 21-2.

22. Martínez-Villén G, Canales V, Hernández-Rossi $A$ et al. Rare manifestation de lésion articu- laire, tendineuse et neurologique par goutte tophacée de la main. Deux cas cliniques. Chir Main, 2007; 26: 55-5.

23. Saffar P. Chondrocalcinosis of the wrist. J Hand Surg Br, 2004; 29: 486-93.

24. Dickinson JC, Kleinert JM. Acute carpal tunnel syndrome caused by a calcified median artery. J Bone Joint Surg Am, 1991; 73: 610-1.

25. Rus A. Compresión nerviosa en la muñeca por músculos aberrantes. Rev Esp Cir Mano, 1996; 51: 65-8.

26. Gwynne Jones DP. Bilateral palmaris profundus in association with bifid median nerve as a cause of failed carpal tunnel release. J Hand Surg Am, 2006; 31: 741-3.

27. Delgado Serrano PJ, Abad Morenilla JM, Dudley Porras AF et al. Síndrome del túnel carpiano asociado a variaciones anatómicas de músculos del antebrazo y mano. Rev Patol Aparato Locom, 2005; 3: 81-6.

28. Bruce Butler JR, Bigley EC. Aberrant index (first) lumbrical tendinous origin associated with carpal tunnel syndrome. J Bone Joint Surg Am, 1971; 53: 160-2.

29. Celester Barreiro G, Castro Río A. Síndrome del túnel carpiano asociado a la existencia de palmar profundo. Aportación de 2 nuevos casos. Rev Iberamer Cir Mano, 2006; 68: 27-30.

30. Sánchez Lorenzo J, Márquez Cañada, López Díaz et al. Compression of the median nerve by an anomalous palmaris longus tendon: a case report. J Hand Surg Am, 1996: 21: 858-60.

31. Beltrán JE, Jimeno-Urban FJ. Variación anatómica del músculo palmar menor. Rev Ortop Traum, 1977; 21 IB: 485-7.

32. Reimann AF, Daseler EH, Anson BJ et al. The palmaris longus muscle and tendon: a study of 1600 extremities. Anat Rec, 1946; 89: 495-505. 
33. Cantero J. Contribution à l'étude des anomalies musculaires de la main et de l'avant-bras (à propos de 58 observations). Tesis doctoral. Universidad de Lausanne (Suiza), 1981.

34. Bauer JM, Trussell JJ. Palmaris profundus causing carpal tunnel syndrome. Orthopedics, 1992; 15: 1348-50.

35. Brones MF, Shaw Wilgis EF. Anatomical variations of palmaris longus causing carpal tunnel syndrome. Plast Reconstr Surg, 1978; 62: 798800.

36. Floyd T, Burger RS, Sciaroni CA. Bilateral palmaris profundus causing bilateral carpal tunnel syndrome. J Hand Surg Am, 1990; 15: 364-6.
37. Fatah MF. Palmaris profundus of Frohse and Frankel in association with a carpal tunnel syndrome. J Hand Surg $\mathrm{Br}$, 1984; 9: 142-4.

38. Mathoulin C, Daïliana Z. Palmaris profundus associé à un syndrome du canal carpien. À propos d'un cas et revue de la literature. La Main, 1998; 3: 261-5.

39. Kanaya K, Wada T, Isogai S et al. Variation in insertion of the abductor digiti minimi: an anatomic study. J Hand Surg Am, 2002; 27: 325-8.

40. Lindau T, Karlsson MK. Complications and outcome in open carpal tunnel release. Ann Chir Main, 1999, 18: 115120.
41. Le Nen D, Rizzo Ch, Hu W et al. Neurolyse du nerf médian au poignet selon la technique endoscopique á deux voies. Ann Chir Main, 1998; 17: 221-9.

42. Benquet B, Fabre T, Durandeau A. Neurolyse du nerf médian au canal carpien par une voie mini-invasive. Á propos d'une série de 138 cas. Chir Main, 2000; 19: 86-93.

43. Dimech C, Laroche D, Vielpeau C. Libération endoscopique du canal carpien. A propos de 100 interventions. Ann Orthop Ouest, 1994; 26: 89-95.

44. González del Pino J, Lovic A, Palazzi $S$ et al. Controversias del destechamiento endoscópico del túnel carpiano. Rev Ortop Traum, 1994; 38 IB: 335-41. 